\title{
Scale-free features of temporal localization of deformation in late stages of creep failure
}

\author{
Tero Mäkinen $\odot,{ }^{1, *}$ Juha Koivisto $\odot,{ }^{1}$ Lasse Laurson, ${ }^{2}$ and Mikko J. Alava ${ }^{1,3}$ \\ ${ }^{1}$ Department of Applied Physics, Aalto University, P.O. Box 11100, FI-00076 Aalto, Espoo, Finland \\ ${ }^{2}$ Computational Physics Laboratory, Tampere University, P.O. Box 692, FI-33014 Tampere, Finland \\ ${ }^{3}$ NOMATEN Centre of Excellence, National Centre of Nuclear Research, A. Soltana 7, 05-400 Otwock-Swierk, Poland
}

(Received 1 June 2020; accepted 21 August 2020; published 18 September 2020)

\begin{abstract}
The last stage of material failure often shows a regime with power-law acceleration as the material lifetime is approached. We study this experimentally in tensile creep with paper samples using digital image correlation. The last, tertiary creep stage exhibits scale-free features in the sample response (strain rate) and its fluctuations. It is accompanied by an increasing localization of strain at the location of final failure. The main features are reproduced by a material model built on a viscoelastic fiber bundle model.
\end{abstract}

DOI: 10.1103/PhysRevMaterials.4.093606

\section{INTRODUCTION}

Material creep under constant loading is accompanied by history effects from accumulation of damage to the buildup of plastic deformation. Material disorder is very important and becomes visible in a heterogeneous deformation field in locally varying strain rates and in the variability of sample lifetimes. Statistical physics models of time-dependent deformation include ingredients such as local thresholds for plastic or elastic deformation bursts, evolving internal stresses and long-range interactions due to the accumulating deformation. These often lead to intermittent avalanche activity.

Finally, materials fail due to the localization of deformation. This happens due to the formation of a shear band or to the nucleation of the final, dominant crack and its growth. These processes are most interesting in time-dependent fracture, where one encounters two main questions: What is the sample lifetime and is it possible to predict it in advance [1-4]? Such models also attempt to describe the typical features of the development of a shear band or the final localization to the region where the material sample fails [5,6]. These general effects, including the avalanches in yielding dynamics and what happens close to the critical yielding transition, are also a rich playground for mesoscopic models $[6,7]$. In recent years, this final regime has attracted plenty of interest. On one hand, a number of experiments point out the so-called power-law acceleration of phenomena when approaching the sample lifetime $t_{c}$. That is, quantities such as acoustic emission [5,8-11] or the strain rate [4,12-14] behave as scale-free power-law functions of $t_{c}-t$. To explain by theoretical arguments and to make careful experimental

\footnotetext{
*Corresponding author; email address: tero.j.makinen@aalto.fi
}

Published by the American Physical Society under the terms of the Creative Commons Attribution 4.0 International license. Further distribution of this work must maintain attribution to the author(s) and the published article's title, journal citation, and DOI. observations of such features thus presents a challenge. One main reason why these findings are of interest is the lifetime prediction of materials from the laboratory to the appearance of a landslide, avalanche, or earthquake.

In this paper, we have performed tensile creep experiments with quasi-2D paper samples with the idea of imaging the sample deformation at a large enough rate to discern the features of the final localization phase. The novelty of this is, among others, in that we look at the local strain rates in this 2D system and in what follows from these experiments. We show that before the final rupture, strain localizes to a single spot. The results on the scaling of strain (-rate) localization indicate that the failure is not a sudden event but a gradually evolving process. Also, either the material does not exhibit the kind of bursty, localized plastic deformation we refer to as a model prediction for disordered materials' yielding, or it happens on such a small scale that it does not leave any clear signatures when studied as herein.

In paper, the time-dependent creep behavior can be divided as is usual in three phases [12] into primary, secondary and tertiary creep. The primary creep regime is described by the Andrade law [15] where the global strain rate $\dot{\epsilon}$ decrease with time $t$ typically follows roughly $\dot{\epsilon} \propto t^{-2 / 3}$, with variations in the exponent value depending on the material. In paper, the secondary creep regime corresponds to logarithmic creep behavior [16], where $\dot{\epsilon} \propto t^{-1}$ [17]. This regime continues until the strain rate minimum is reached, which for the copy paper and geometry we use in our experiments has been found to happen at $t=0.83 \times t_{c}$ where $t_{c}$ denotes the time of the failure of the sample [12]. This relation is known in material science as the Monkman-Grant relationship [18] and it implies that the creep responses of different samples can be collapsed to a master curve of the form $\dot{\epsilon}=f\left(t / t_{c}\right) \min \dot{\epsilon}$, with some shape function $f$, as the response depends only on the minimum strain rate and the failure time [19]. After the minimum, there is the regime that we are interested in, the tertiary creep regime, where the strain rate increases rapidly $[12,20,21]$, which implies strain softening. This increase finally leads to the sample failure at time $t_{c}$. 
(a)

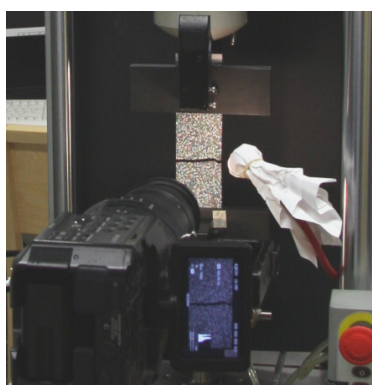

(b)
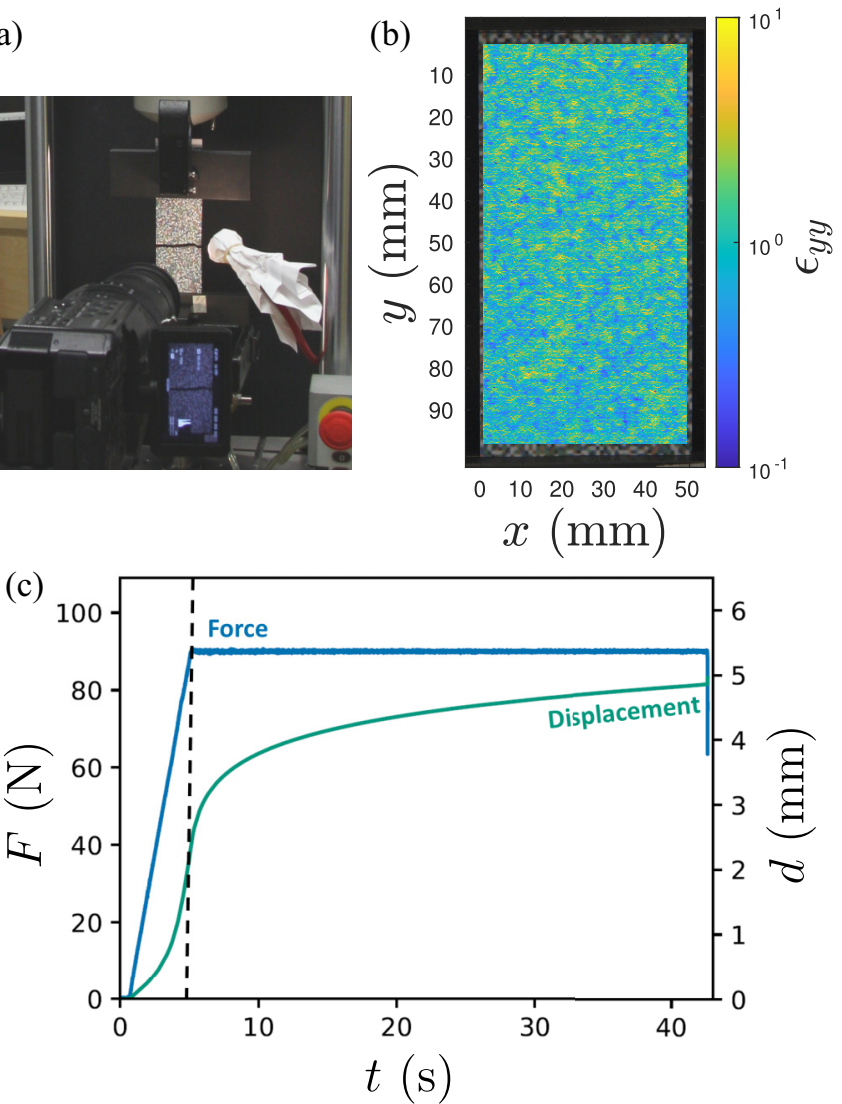

FIG. 1. (a) Picture of the experiment. The $4 \mathrm{k}$ videocamera is at the front of the Instron E1000 tensile testing machine. The lighting is provided by a led light with paper light diffusor. The sample is a $50 \times 100 \mathrm{~mm}^{2}$ paper sheet with grammage of $75 \mathrm{~g} / \mathrm{m}^{2}$ printed with colored random speckle pattern. One dot is approximately 32 pixels in diameter so $1 \mathrm{~mm}$ in nature. (b) Strain map $8 \%$ before sample failure overlaid on top of the reference image of the sample. The areas of roughly uniform strain which are around 2-3 mm correspond to the paper floc size. (c) Raw data from the tensile testing machine shows an example of the stress-controlled experiment with 4.75 second initial increasing stress ramp followed by a constant $F=95 \mathrm{~N}$ loading until the sample breaks at $t=42.5 \mathrm{~s}$ (blue, right axis). The measured total displacement $d$ is a typical creep curve with decreasing strain rate (derivative) until few seconds before the end (green, right axis).

The behavior of the local fluctuations in the strain rate (quantified by the standard deviation of the local strain rates in the vertical direction $\Delta \dot{\epsilon}_{y y}$ ) is known to decay as a power-law $\Delta \dot{\epsilon}_{y y} \propto t^{-\gamma}$ [22] in the initial two regimes. The exponent $\gamma$ is smaller than what is found for the global strain rate $(\gamma \approx 0.5)$. So as the decay is slower, the fluctuations become more important as the experiment progresses. We next turn our focus on the final, tertiary phase and the statistical deformation signals there.

\section{METHODS}

The experimental setup is shown in Fig. 1(a). The Instron E1000 tensile testing machine created a constant loading $F$ to the dotted sample at the center and recorded the displacement

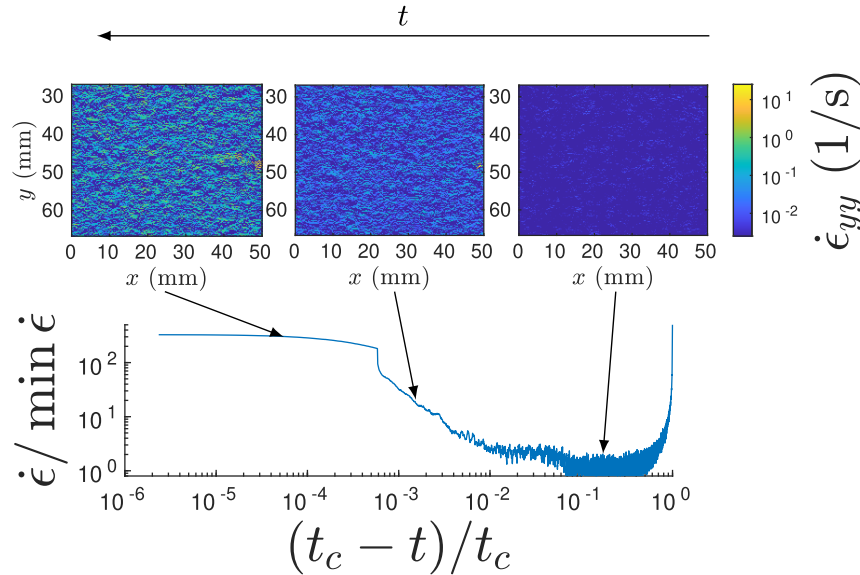

FIG. 2. An example experiment showing the behavior of the global strain rate $\dot{\epsilon}$ of the sample as a function of the normalized time before failure $\left(t_{c}-t\right) / t_{c}$ (bottom), where $t_{c}$ is the failure time of the sample. The vertical strain rate component $\dot{\epsilon}_{y y}$ (top) obtained from DIC is shown at a few selected points in time corresponding to the locations on the strain rate curve shown by the arrows. Strain rate localization into a single region around $y=50 \mathrm{~mm}$ and $x=50 \mathrm{~mm}$ can be seen well before the failure.

$d$ of the piston. The global strain was calculated from the displacement as $\epsilon=d / h$, where $h$ is the initial height of the specimen. The Sony PXW-FS5 4k video camera recorded images of the samples with high resolution $(30 \mu \mathrm{m} / \mathrm{pixel})$ to a $25 \mathrm{~Hz}$ video stream. The paper sample was a $50 \mathrm{~mm} \times$ $100 \mathrm{~mm}$ copy paper sample with a grammage of $75 \mathrm{~g} / \mathrm{m}^{2}$. The sample was printed with a randomly colored speckle pattern where the $1 \mathrm{~mm} \times 1 \mathrm{~mm}$ dots are in a square grid. The tensile testing machine created a constant load that was varied between experiments from 90 to $100 \mathrm{~N}$ with an initial ramp performed at a rate of $20 \mathrm{~N} / \mathrm{s}$. The loading protocol is depicted in Fig. 1(c). The results presented here consist of six experiments with lifetimes varying from $45 \mathrm{~s}$ to $1380 \mathrm{~s}$; these are picked out of a larger set of experiments based on the criterion that they have a sample lifetime much larger than then ramp-up time to the constant stress level.

The digital image correlation (DIC) is performed using the NCORR [23] software which calculates the local displacements $\boldsymbol{u}=(u, v)$ with respect to the first image using a circular region of interest with a radius of $1.0 \mathrm{~mm}$ and placing the regions of interest every four pixels (corresponding to a spacing of $0.1 \mathrm{~mm}$ ). One should compare this scale to the typical structural scale in paper, that of flocs (the clusters of fibers that form in papermaking and show up as the evident cloudiness in the paper structure). That scale is typically of the order of $2 \mathrm{~mm}$ [17]. The local strain component in the loading direction is calculated from these displacements as $\epsilon_{y y}=\frac{\partial v}{\partial y}$ [see Fig. 1(b)] and the strain rate component $\dot{\epsilon}_{y y}$ is then obtained via numerical differentiation of successive strain maps. These local strain rate maps are shown in Fig. 2. The DIC calculations are performed for images corresponding to approximately 250 points in time (concentrated to the end of the experiment) in each experiment, or the last $10 \mathrm{~s}$ in time.

As the accuracy of the DIC calculations is roughly $10 \%$ of the pixel size, the noise floor for strain calculations would be 
around 0.03 . The strain-rate resolution varies according to the time interval between the images considered but ranges from $10^{-3} \mathrm{~s}^{-1}$ (when the deformation is the slowest) to $0.75 \mathrm{~s}^{-1}$ (right before failure). We note that DIC strain maps should also reflect localized deformation activity, since measuring over several avalanches happening in windows of time does not hide immediately any power-law-like distributions possibly present on the microscopic scale [24].

Another way to characterize the strain rate localization is to define a threshold (for example one standard deviation above the mean $\left.\dot{\epsilon}_{\text {thres }}=\left\langle\dot{\epsilon}_{y y}\right\rangle+\Delta \dot{\epsilon}_{y y}\right)$ and to look at the spatial distribution of points above this threshold. One can then define the localization coefficient [6]

$$
\eta_{y}=1-\left(\frac{\Delta y}{\Delta_{0} y}\right)^{2}
$$

where $\Delta y$ is the standard deviation of the $y$ coordinates of the locations where $\dot{\epsilon}_{y y}>\dot{\epsilon}_{\text {thres }}$ and $\Delta_{0} y=L_{y} / \sqrt{12}$ the standard deviation of randomly placed locations.

\section{Serial fiber bundle model}

To model this behavior, we resort to a serial viscoelastic fiber bundle model (SFBM) [12,25-27], used earlier to analyze the Monkman-Grant relation [12] which consists of $N_{s}$ serial layers of fiber bundles with $N_{p}$ fibers each. The viscoelastic fibers are modeled as Kelvin-Voigt elements $\sigma=$ $\beta \dot{\epsilon}+E \epsilon$, where $\sigma$ is the constant load, $\beta$ the damping constant, and $E$ the Young modulus. Here we set $\beta=E=1$ for simplicity. The fibers have a failure strain which is drawn from a distribution $p\left(\epsilon_{c}\right)$, which we have here chosen to be uniform between 0 and 1 . If a fiber fails, then the load is equally distributed among the remaining intact fibers within the same bundle (global load sharing). Hence, the time evolution of each of the $N_{s}$ bundles in series is described by $\sigma_{0} /[1-P(\epsilon)]=\beta \dot{\epsilon}+E \epsilon$, where $P$ is the cumulative distribution of failure thresholds of the given bundle.

The simulations are performed by setting the external load $\sigma_{0}$ to be slightly above the critical value above which one of the bundles will eventually fail. This, together with the small random fluctuations from bundle to bundle in the bundlespecific failure strain distributions results in a distribution of failure times for the $N_{s}$ different bundles in series. The simulation considers the simulated sample to fail when the first of the serial layers fails, which happens when all fibers in this layer have failed. Here we have chosen $N_{b}=12800$ and $N_{s}=40$; the latter choice is motivated by the observation that in our experiments on paper samples the sample height corresponds to roughly 40 times the floc size of paper.

\section{RESULTS}

Looking at the strain maps [Fig. 1(b)] produced by DIC, one can observe areas of roughly uniform strain that are around $2-3 \mathrm{~mm}$ in size. This size is larger than the size of the dots printed to the sample and larger than the region of interest in the DIC calculations. The main features of paper structure are the flocs - the aggregates of fibers produced in the papermaking process - and the size of these areas of uniform strain correspond to the floc size of the paper [17]. (a) (b)

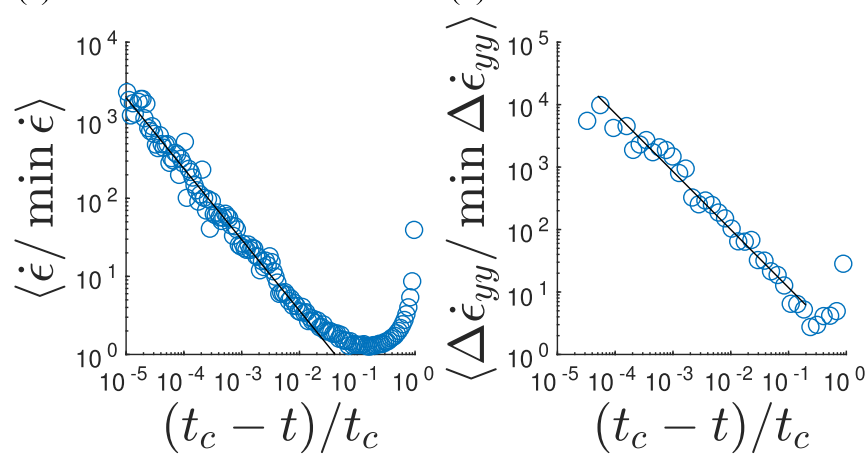

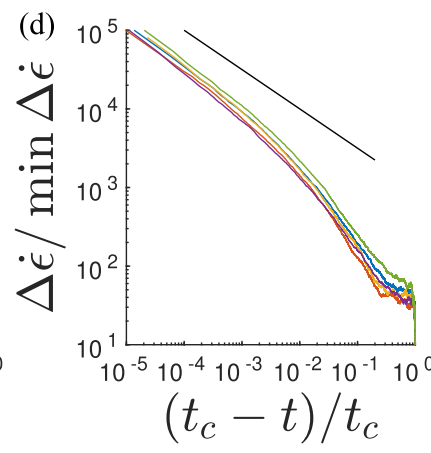

FIG. 3. (a) The average behavior of the global strain rate $\dot{\epsilon}$ (obtained from the tensile testing machine data) as a function of the normalized time before failure $\left(t_{c}-t\right) / t_{c}$. The black line represents a power-law fit $\left(t_{c}-t\right)^{-0.91}$. (b) The standard deviation of the vertical component of the strain rate obtained from the DIC. The black line represents a power-law fit $\left(t_{c}-t\right)^{-0.93}$. (c) The strain rate as a function of the normalized time before failure from the SFBM. The different lines correspond to different realizations of the simulation. The black line represents a power-law $\left(t_{c}-t\right)^{-1 / 2}$. (d) The standard deviation of the strain rates of the different bundles in the SFBM. The different lines correspond to different realizations of the simulation. The black line represents a power-law $\left(t_{c}-t\right)^{-1 / 2}$.

We reproduce the earlier results in that the experiments reach a strain rate minimum on average at $t=0.83 \times t_{c}$ (the Monkman-Grant relationship) and after that the strain rate increases (see bottom part of Fig. 2). Looking at the strain rate maps at corresponding times (see top part of Fig. 2), one can observe localization of the strain rate into a single location in this tertiary creep regime. This location matches with the eventual one for failure.

When looking at the average behavior of the global strain rate $\dot{\epsilon}$, we first normalize it with its minimum value $\min \dot{\epsilon}$ for each experiment. After that, we see a power-law divergence $\langle\dot{\epsilon} / \min \dot{\epsilon}\rangle \propto\left(t_{c}-t\right)^{-b}$ with $b=0.91 \pm 0.04$ [see Fig. 3(a)] as failure is approached. This power-law spans around three decades in time (starting around 1\% before the sample failure) and is observed right up to the limit of the time resolution of our experimental setup.

As also the local strain rates $\dot{\epsilon}_{y y}$ are measured over the whole sample, one can quantify the deformation behavior using their statistics. Looking at the standard deviation of the local strain rate over the whole sample $\Delta \dot{\epsilon}_{y y}$ shows that this quantity also increases while approaching failure, indicating growing fluctuations. In fact, after normalizing it with 
the minimum value min $\Delta \dot{\epsilon}_{y y}$ for each experiment (similarly to the global strain rate), we observe a power-law divergence $\left\langle\Delta \dot{\epsilon}_{y y} / \min \Delta \dot{\epsilon}_{y y}\right\rangle \propto\left(t_{c}-t\right)^{-c}$ with $c=0.93 \pm 0.06$ [see Fig. 3(b)]. Again, this power-law spans around three decades, starting slightly earlier than the power law of global strain rate, around the global strain rate minimum. The slight dip in the curve with the smallest values of $\left(t_{c}-t\right) / t_{c}$ is probably due to DIC algorithm's inability to follow such large strain rates localized in such small area (these correspond only to the last or second-to-last images before failure).

The time-dependent strain rate obtained from the SFBM shows a similar strain rate minimum at $t=0.83 \times t_{c}$ [see Fig. 3(c)] as the experiments; this has been demonstrated to be a geometry-controlled effect such that the time of the strain rate minimum can be tuned by changing $N_{s}$ [12]. After that, the strain rate increases and, similar to the experiments, a power-law divergence $\dot{\epsilon} / \min \dot{\epsilon} \propto\left(t_{c}-t\right)^{-b_{\text {SFBM }}}$ is seen. Here the exponent $b_{\mathrm{SFBM}}$ is smaller than that in our paper experiments. For the SFBM, we find a value close to $1 / 2$, as found before for individual viscoelastic fiber bundles [26]. The power-law regime starts around $1 \%$ before failure, again similar to the experiments.

Also the standard deviation (which here means the standard deviation between the serial layers) of the strain rate, $\Delta \dot{\epsilon}(t)$, increases as the failure is approached [see Fig. 3(d)]. Again close to failure a power-law divergence with an exponent around the same value as for the mean rate (i.e., 1/2) is found. A possible interpretation of the standard deviation exponent equaling that describing the mean rate is due to deformation localization close to final failure, which in the SFBM is a purely statistical effect (one of the bundles happens to fail before the others): In a typical case, the final divergence of the strain rate is dominated by the local strain rate at the location of the eventual failure.

As the final failure is brought up by a crack running roughly horizontally through the sample, the strain-rate maps can be reduced to one dimension to better grasp the information about the localization. This is done by averaging the maps along the $x$ direction, yielding $\left\langle\dot{\epsilon}_{y y}\right\rangle_{x}$, which is a function of only the vertical coordinate $y$. Plotting this as a function of the normalized time before failure $\left(t_{c}-t\right) / t_{c}$ [see Fig. 4(a)], one can clearly observe the localization into a single spot. Even by eye this is can be seen around $5 \%$ before failure, so slightly later than the start of the observed power-law divergence of the standard deviation of the local strain rate. Using thresholding to define and measure localization would result in the same conclusion. The width of this spot again corresponds to the structural scale, floc size, being around 2-3 $\mathrm{mm}$.

The same can naturally be done to the strain $\epsilon_{y y}$ yielding $\left\langle\epsilon_{y y}\right\rangle_{x}$ which behaves in a similar way [see Fig. 4(b)]. In this case, again plotting against $\left(t_{c}-t\right) / t_{c}$, the localization is less clear, but observable by eye around $0.2 \%$ before failure. Notably, in this example, one can also observe some localization of strain outside the final failure site at $y=50 \mathrm{~mm}$ (namely, at $y=38 \mathrm{~mm}$ ). Similar features also come out of the fiber bundle model, see Figs. 4(c) and 4(d). The agreement is of course only qualitative. We have computed various correlation functions of the strains and strain rates as a function of time and for two-point quantities as a function of spatial distance. They agree with Fig. 4: The correlations that build up are
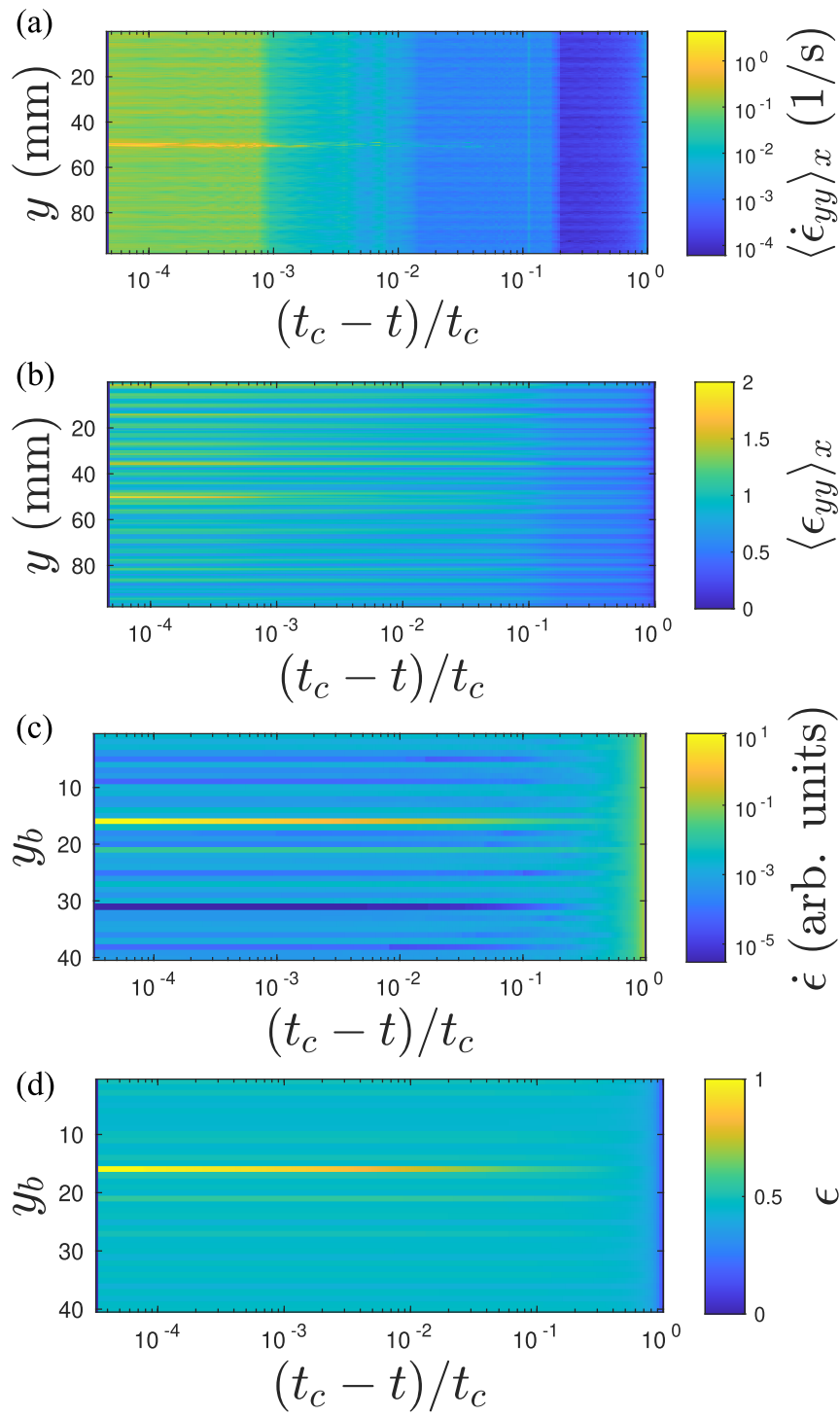

FIG. 4. (a) An example experiment showing the behavior of the strain rate $\dot{\epsilon}_{y y}$ averaged over the width of the sample $\left\langle\dot{\epsilon}_{y y}\right\rangle_{x}$ as a function of the normalized time before failure. The localization into a single eventual failure site at around $y=50 \mathrm{~mm}$ is clearly visible $5 \%$ before failure which is slightly later than the onset of the power-law increase of $\Delta \dot{\epsilon}_{y y}$ seen in Fig. 3. (b) The same plot as (a) but for the strain $\epsilon_{y y}$. The localization is still obvious but the starting point is less clear. In addition to the final failure site at around $y=50 \mathrm{~mm}$, one can see some minor strain localization at $y=38 \mathrm{~mm}$. (c) A realization of the SFBM simulation showing the behavior of the strain rates $\dot{\epsilon}$ at different bundles $y_{b}$ as a function of the normalized time before failure. (d) The same plot as $\mathrm{c}$ but for the strain $\epsilon$.

due to localization in the $y$ direction. At short ranges, up to one millimeter, some features can be seen that originate from the disordered structure of paper but they do not evolve much in tertiary creep. In earlier work [28], it was found that in contrast to the primary creep phase where the relative importance of fluctuations grows, such correlations might be interesting.

These results are also reproduced by the localization measure. What we see at first glance (see the inset of Fig. 5) seems 


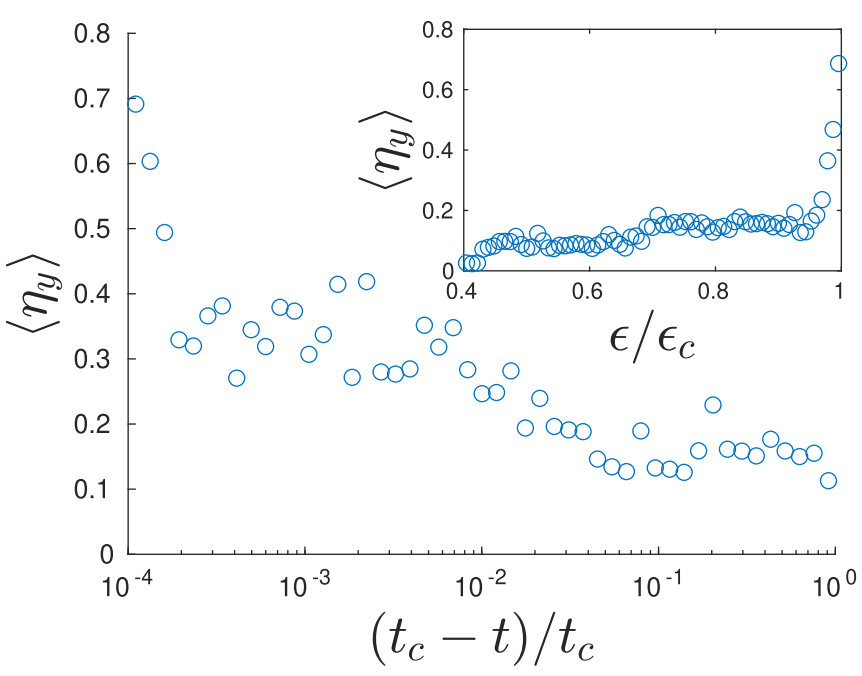

FIG. 5. The average behavior of the localization coefficient $\eta_{y}$ as a function of the normalized time before failure. The inset shows the same behavior as a function of normalized strain.

to be a behavior rather independent of $t_{c}-t$ until almost the very end of the experiment, even though the main figure gives the impression that there is a logarithmic, slow increase until the same final abrupt localization. This measure seems to indicate that both the integrated strain and the strain-rate maps - the latter produce, formally speaking, the former upon integration in time - do not show any signatures of the bursty avalanche activity one might expect. Also looking at the distributions of the local strain rates, following their development over time by histograms does not reveal any signatures of the fat-tailed distributions one would expect from avalanche activity. In plain terms, if the sample response would arise from avalanches these would become visible locally in the strain rates (strain over the DIC time window).

\section{CONCLUSIONS}

We have studied the localization of deformation close to failure in tensile creep of a disordered quasi-2D material using DIC. The main result is that the localization becomes visible after the strain-rate minimum of the global strain-rate curve in tertiary creep. This localization can be seen first in the standard deviation of the local strain rate (right after the strain rate minimum, so $17 \%$ before failure), later by eye in the local strain rate averaged over the horizontal direction (5\% before failure), and finally by eye in the local strain averaged over the horizontal direction $(0.2 \%$ before failure). In other words, a band of roughly constant width becomes visible in the strain analysis.

The associated quantities, such as the global strain rate $\dot{\epsilon}$ and the standard deviation of the local strain rate in the vertical direction $\Delta \dot{\epsilon}_{y y}$, show scale-free behavior. This is seen in the form of a power-law divergence as the failure is approached. In both cases, the exponent is close to unity so the divergence is close to $\left(t_{c}-t\right)^{-1}$. This is in contrast to what happens to these quantities in primary and secondary creeps, where their values are such that the fluctuations are more prominent as they decay more slowly than the creep rate itself [28].

This behavior can be reproduced qualitatively by a SFBM where the bundles can be thought to correspond to representative volume elements of the size of individual flocs in paper. The similar power-law exponents for the divergences of the strain rate and the local deviations in the strain rate might imply that the material response of a single bundle (or in experiments failure location) dominates the divergence. As the experiments can be modeled using this type of model, where the local damage developments are not correlated, this would imply that the deformation localization in paper is also not correlated in the way it would be if it would the result of avalanching dynamics of local deformation bursts. This is in line with the fact that the localization measure shows no signs of correlated localized avalanche activity. The final failure is due to the appearance of a local spot of fast deformation at the $t_{c}$ or close. Due to the general nature of the model used to compare with experiments, we would expect that there are other materials which belong to the same universality class.

Future work, in addition to testing other materials, could include a more detailed version of the SFBM with crack nucleation built in to quantitatively reproduce the divergence laws and to explore the final failure. It would also be of interest to look at geometry effects: long and narrow versus short and broad samples.

\section{ACKNOWLEDGMENTS}

M.J.A. acknowledges support from the Academy of Finland (Center of Excellence Programs No. 278367 and No. 317464) and the European Union Horizon 2020 research and innovation program under Grant Agreement No. 857470 and from European Regional Development Fund via the Foundation for Polish Science International Research Agenda PLUS program Grant No. MAB PLUS/2018/8. J.K. acknowledges funding from Academy of Finland (308235) and Business Finland (211715). L.L. acknowledges the support of the Academy of Finland via the Academy Project COPLAST (Project No. 322405). The authors acknowledge the computational resources provided by the Aalto University School of Science "Science-IT" project, as well as those provided by CSC - IT Center for Science (Finland).
[1] B. Voight, Science 243, 200 (1989).

[2] D. Sornette, Proc. Natl. Acad. Sci. 99, 2522 (2002).

[3] A. F. Bell, M. Naylor, M. J. Heap, and I. G. Main, Geophys. Res. Lett. 38, L15304 (2011).
[4] S.-W. Hao, B.-J. Zhang, J.-F. Tian, and D. Elsworth, J. Geophys. Res. Solid Earth 119, 1942 (2014).

[5] S. Lennartz-Sassinek, I. G. Main, M. Zaiser, and C. C. Graham, Phys. Rev. E 90, 052401 (2014). 
[6] D. F. Castellanos and M. Zaiser, Phys. Rev. Lett. 121, 125501 (2018).

[7] A. Nicolas, E. E. Ferrero, K. Martens, and J.-L. Barrat, Rev. Mod. Phys. 90, 045006 (2018).

[8] A. Guarino, S. Ciliberto, A. Garcimartın, M. Zei, and R. Scorretti, Eur. Phys. J. B 26, 141 (2002).

[9] G. F. Nataf, P. O. Castillo-Villa, P. Sellappan, W. M. Kriven, E. Vives, A. Planes, and E. K. Salje, J. Phys. Condens. Matter 26, 275401 (2014).

[10] J. Vasseur, F. B. Wadsworth, Y. Lavallée, A. F. Bell, I. G. Main, and D. B. Dingwell, Sci. Rep. 5, 13259 (2015).

[11] X. Liu, J. Yu, Y. Zhu, W. Yao, and Y. Lai, Front. Earth Sci. 8, 58 (2020).

[12] J. Koivisto, M. Ovaska, A. Miksic, L. Laurson, and M. J. Alava, Phys. Rev. E 94, 023002 (2016).

[13] V. Grenard, T. Divoux, N. Taberlet, and S. Manneville, Soft Matter 10, 1555 (2014).

[14] B. Saint-Michel, T. Gibaud, and S. Manneville, Soft Matter 13, 2643 (2017).

[15] E. N. d. C. Andrade, Proc. R. Soc. London 84, 1 (1910).

[16] A. Cottrell, Philos. Mag. Lett. 75, 301 (1997).
[17] M. Alava and K. Niskanen, Rep. Prog. Phys. 69, 669 (2006).

[18] F. C. Monkman and N. J. Grant, Proc. ASTM 56, 593 (1956).

[19] S. Biswas, D. F. Castellanos, and M. Zaiser, arXiv:2005.03514.

[20] H. Nechad, A. Helmstetter, R. El Guerjouma, and D. Sornette, Phys. Rev. Lett. 94, 045501 (2005).

[21] M. Leocmach, C. Perge, T. Divoux, and S. Manneville, Phys. Rev. Lett. 113, 038303 (2014).

[22] J. Rosti, J. Koivisto, L. Laurson, and M. J. Alava, Phys. Rev. Lett. 105, 100601 (2010).

[23] J. Blaber, B. Adair, and A. Antoniou, Exp. Mech. 55, 1105 (2015).

[24] R. Kokkoniemi, A. Miksic, M. Ovaska, L. Laurson, and M. J. Alava, J. Stat. Mech. (2017) 073401.

[25] M. J. Alava, P. K. Nukala, and S. Zapperi, Adv. Phys. 55, 349 (2006).

[26] R. C. Hidalgo, F. Kun, and H. J. Herrmann, Phys. Rev. E 65, 032502 (2002).

[27] F. Kun, R. C. Hidalgo, H. J. Herrmann, and K. F. Pál, Phys. Rev. E 67, 061802 (2003).

[28] L. Laurson, J. Rosti, J. Koivisto, A. Miksic, and M. J. Alava, J. Stat. Mech. (2011) P07002. 